\title{
Omnidirectional Guided Wave PWAS Phased Array for Thin-wall Structure Damage Detection
}

\author{
Lingyu $\mathrm{Yu}$, \\ Mechanical Engineering Department, University of South Carolina \\ Columbia, SC 29208,yu3@engr.sc.edu \\ Victor Giurgiutiu, \\ Mechanical Engineering Department, University of South Carolina \\ Columbia, SC 29208, giurgiut@engr.sc.edu \\ James R Kendall, \\ Michoud Operation, Lockheed Martin Space Systems Company \\ James.R.Kendall@maf.nasa.gov
}

\begin{abstract}
Widely used 1-D guided wave phased arrays can only scan within $0^{\circ} \sim 180^{\circ}$ range due to the intrinsic structural limitation. To overcome this limitation, multiple installations are unavoidable. However, by using 2-D configuration phased arrays, large structural interrogation can be conducted from a single location with a single scanning covering the entire $0^{\circ} \sim 360^{\circ}$ range through omnidirectional beamforming. Based on the developed generic PWAS Lamb wave phased array beamforming formula, this paper aims to bring up the practical implementation of an $8 \times 8$ PWAS array and present analytical and experimental results concerning the omnidirectional damage detection ability.

The theoretical beamforming of 2-D rectangular PWAS configuration will be presented at first based on the generic PWAS phased array beamforming formulation. Then, an $8 \times 8$ rectangular PWAS array is installed on the target aluminum specimen. Laboratory experiment is set up next and conducted various crack damage detection. Specimens include: (1) a single crack positioned at $90^{\circ}$; (2) a single crack positioned at $270^{\circ}$; (3) a single hole positioned at $\left.180^{\circ} ; 4\right)$ a crack at $90^{\circ}$ and a hole at $180^{\circ}$, respectively. The experiments will demonstrate the $360^{\circ}$ damage finding ability of the 2-D PWAS array and the multiple damage detection ability as well. We finally end up with discussions on the practical application and come up with plans for future work on various 2-D array developments.
\end{abstract}

Keywords: guided waves, phased array, omnidirectional beamforming, piezoelectric wafer active sensor, PWAS, piezoelectric, damage detection, crack

\section{INTRODUCTION}

Guided wave PWAS phased arrays are a method of creating a virtual beam that sweeps the horizon through delay-and-sum beamforming by using a group of permanently attached (embedded) piezoelectric wafer active sensors (PWAS) [1]. Details of PWAS operation principles and the use of PWAS for SHM can be found in [1]. Similarly to conventional ultrasonic transducers, PWAS can be used in pitch-catch, pulse-echo, phased array, and other NDE modes. In the pulse-echo method, a PWAS transducer attached to the structure acts as both transmitter and detector of guided Lamb waves traveling in the structure. The use of PWAS phased array enhances the pulse-echo mode by strengthening the echo signal and enabling directional scanning capability.

PWAS phased arrays work best with single-mode non-dispersive Lamb waves. However, at least two Lamb modes, A0 and S0, exist simultaneously for many SHM approaches using guided waves, where the product of the wave frequency and structure thickness falls in the range of $0 \sim 1 \mathrm{MHz} \cdot \mathrm{mm}$. At these values, the A0 mode is highly dispersive, while the S0 mode is almost non-dispersive and hence better suited for crack detection with the pulse-echo method. Hence, the critical issue becomes exciting only non-dispersive S0 Lamb mode. In another word, we need to tune the excitation at a frequency where S0 Lamb mode dominates and A0 Lamb mode is minimized. Giurgiutiu showed that given the PWAS length $2 a$, plate thickness $2 d$, and material properties $\mu$ and $\lambda$, it is possible to find frequencies at which only one mode is excited (tuning frequency) [2]. A plane-strain analysis of the PWAS-structure interaction using the space-domain Fourier analysis has been developed to illustrate the principle of PWAS Lamb wave mode tuning. That is to say, a selected Lamb mode 
can be tuned by choosing the appropriate frequency and PWAS dimensions. A plot of the strain solution in the $0 \sim 700 \mathrm{kHz}$ bandwidth for an aluminum plate of $1 \mathrm{~mm}$ thickness installed with $7 \mathrm{~mm}$ round PWAS is presented in Figure 1a while Figure 1b gives the group velocity. In Figure 1a, a S0 tuning frequency around $300 \mathrm{kHz}$ can be found, where the amplitude of the A0 mode is minimized while that of the S0 mode is still strong. Therefore, we have tuning of the S0 mode and are able to reject the A0 mode. These predictions have been extensively verified experimentally [3]. We see that Lamb wave tuning offers considerable advantages, allowing us to select Lamb waves that are most appropriate for the particular application being considered.
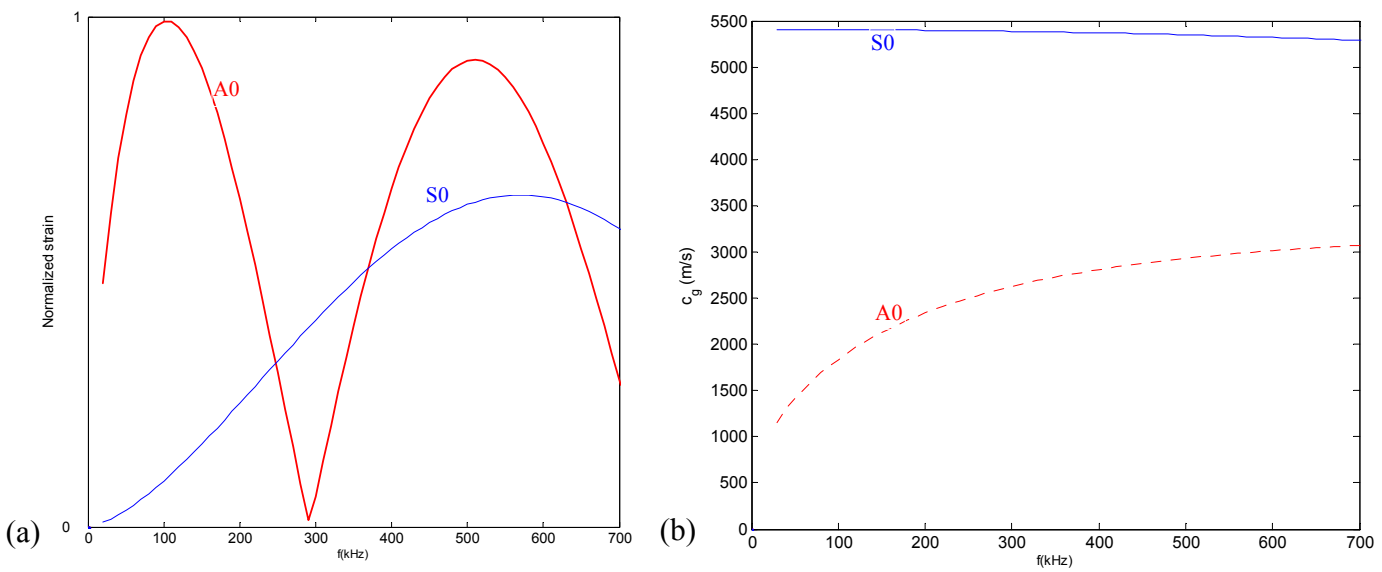

Figure 1 Lamb wave S0 mode tuning on a 1-mm thick aluminum plate using 7-mm round PWAS within 0 700 kHz range. (a) strain curve; (b) group velocity (dispersion curves)

\section{2-D PWAS PHASED ARRAY BEAMFORMING}

The ultrasonics-based active SHM method uses PWAS to transmit and receive Lamb waves in a thin-wall structure. The principle of operation of PWAS phased array is derived from two general principles: (1) The principle of guided Lamb wave generation and sensing with PWAS in pulse echo pattern; (2) The principles of conventional phased-array radar. In this research, we developed the PWAS phased array beamforming formulas based on the conventional delay-and-sum beamforming and the full wave paths for delays calculation. The derived algorithm can be applied to any fields in the interrogated structures. The delay-and-sum beamforming consists of two steps:

- Applying delay $\Delta_{m}$ and weights $w_{m}$ to the propagating wave from the $m^{\text {th }}$ PWAS, $f\left(\vec{r}_{m}, t\right)$;

- Summing up the output signals of the total of $M$ PWAS.

This two-step procedure can be mathematically expressed as

$$
z(\vec{r}, t)=\sum_{m=0}^{M-1} w_{m} f\left(\vec{r}_{m}, t-\Delta_{m}\right)
$$

where $f\left(\vec{r}_{m}, t\right)$ is the wave coming from the $m^{\text {th }}$ PWAS source. The delays can be adjusted as to focus the array's maximum output beam on a particular propagating direction while the weighting factors can be used for further beam enhancement such as changing the beams' shape or reducing sidelobe levels [4].

To find out the generic beamforming formulas, we assume:

- All the elements in the array lie in the same plane

- All the elements behave as point-wise sources and receivers (omnidirectional active sensors)

- Monochromatic excitation and reception regardless of the dispersion of guided wave applies

- Simultaneous and uniform excitation

The nondispersion assumption results in a constant speed $c$ in isotropic material. We will find the beamforming at a point $P(r, \phi)$ by an arbitrary array consisting of a total of $M$ PWAS located at $\left\{\vec{s}_{m}\right\}, m=0,1, \ldots M-1$. The 
coordinate systems is defined by using the array phased center where the origin coincides with the phased center $\frac{1}{M} \sum \vec{s}_{m}=0$. As illustrated in Figure 2, the target $P(r, \phi)$ is $\vec{r}_{m}$ away from the $m^{\text {th }}$ PWAS located at $\vec{s}_{m}$. We define $\vec{\xi}$ as the unit direction vector from origin $O$ to the $\operatorname{target} P$ and $\vec{\xi}_{m}$ as the unit direction vector from $m^{\text {th }}$ PWAS to $P$.

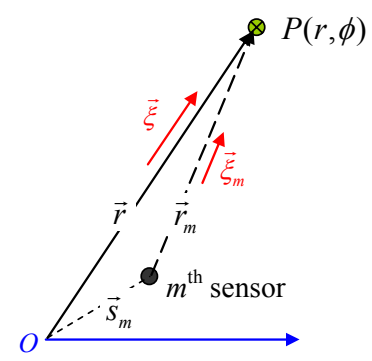

Figure 2 Geometrical schematic of an $M$-PWAS array with a target located at point $P$

For a single tone, outgoing radial mode wave $f\left(\vec{r}_{m}, t\right)$, its wave front at the $\operatorname{target} P$ is in the form

$$
f(\vec{r}, t)=\frac{A}{\sqrt{|\vec{r}|}} e^{j(\omega t-\vec{k} \cdot \vec{r})}
$$

with $\vec{k}$ as the wave number, $\vec{k}=\vec{\xi} \cdot \omega / c$, and $\omega$ as the angular excitation frequency. To facilitate the calculation, following notations are defined

$$
\begin{gathered}
\vec{\xi}=\frac{\vec{r}}{r}, r=|\vec{r}|, \vec{k}=\vec{\xi} \cdot \frac{\omega}{c} \\
\vec{r}_{m}=\vec{r}-\vec{s}_{m}, r_{m}=\left|\vec{r}_{m}\right|, \vec{\xi}_{m}=\frac{\vec{r}_{m}}{\left|\vec{r}_{m}\right|}, \vec{k}_{m}=\vec{\xi}_{m} \cdot \frac{\omega}{c}
\end{gathered}
$$

where $\vec{k}_{m}$ is the wave number of the wave propagating in the direction of $\vec{\xi}_{m}$. Using notations in (3) and (4), wave front at the target $P$ from $m^{\text {th }}$ PWAS is

$$
f\left(\vec{r}_{m}, t\right)=\frac{A}{\sqrt{r_{m}}} e^{j\left(\omega t-\vec{k}_{m} \cdot \vec{r}_{m}\right)}
$$

And the synthetic wave front at $P$ from the total of $M$ PWAS is

$$
z(\vec{r}, t)=\sum_{m=0}^{M-1} w_{m} f\left(\vec{r}_{m}, t\right)=\sum_{m=0}^{M-1} w_{m} \frac{A}{\sqrt{r_{m}}} e^{j\left(\omega t-\vec{k}_{m} \cdot \vec{r}_{m}\right)}
$$

The synthetic wave front in Equation (6) can be re-written as

$$
z(\vec{r}, t)=f\left(t-\frac{r}{c}\right) \cdot \sum_{m=0}^{M-1} w_{m} \frac{1}{\sqrt{r_{m} / r}} e^{j \omega\left(1-\frac{r_{m}}{r}\right) / c}
$$

We normalized the quantity $r_{m}$ by the quantity $r, \tilde{r}_{m}=r_{m} / r$, resulting

$$
z(\vec{r}, t)=f\left(t-\frac{r}{c}\right) \cdot \sum_{m=0}^{M-1} w_{m} \frac{1}{\sqrt{\tilde{r}_{m}}} e^{j \omega\left(1-\tilde{r}_{m}\right) / c}
$$

In Equation (8), we notice that the synthetic signal $z(\vec{r}, t)$ is fully determined by the second multiplier (summation), which depends on the weights $\left\{w_{m}\right\}$ and the normalized PWAS locations $\left\{\tilde{r}_{m}\right\}$. We call the 
second multiplier as the beamforming factor (represented as $B F$ ). $B F$ is given as

$$
B F\left(w_{m}, \tilde{r}_{m}\right)=\sum_{m=0}^{M-1} w_{m} \frac{1}{\sqrt{\tilde{r}_{m}}} e^{j \omega\left(\frac{1-\tilde{r}_{m}}{c}\right)}
$$

To steer the output wave front $z(\vec{r}, t)$ at certain direction $\phi_{0}$, i.e., to reinforce the wave in the desired direction with respect to waves propagating in other directions, the delays $\left\{\Delta_{m}\left(\phi_{0}\right)\right\}$ are introduced, i.e.,

$$
B F\left(w_{m}, \tilde{r}_{m}\right)=\sum_{m=0}^{M-1} w_{m} \frac{1}{\sqrt{\tilde{r}_{m}}} e^{j \omega\left(\frac{1-\tilde{r}_{m}}{c}-\Delta_{m}\left(\phi_{0}\right)\right)}
$$

Equation (10) shows that the maximum beamforming $B F\left(w_{m}, \tilde{r}_{m}\right)$ is expected if the exponential parts equal to one, $\left(1-\tilde{r}_{m}\right) / c-\Delta_{m}\left(\phi_{0}\right)=0$, or, $\exp \left\{\left(1-\tilde{r}_{m}\right) / c-\Delta_{m}\left(\phi_{0}\right)\right\}=1$. Hence, the proper delay applied to the $m^{\text {th }}$ PWAS is

$$
\Delta_{m}\left(\phi_{0}\right)=\frac{1-\tilde{r}_{m}}{c}
$$

Then the beamforming factor at this particular direction $\phi_{0}$ becomes

$$
B F\left(w_{m}, \tilde{r}_{m}, \phi_{0}\right)=\sum_{m=0}^{M-1} w_{m} \frac{1}{\sqrt{\tilde{r}_{m}}}
$$

Equation (12) shows further manipulation to shape the maximum beam at the desired direction $\phi_{0}$ can be achieved by adjusting the weighting factor $\left\{w_{m}\right\}$. One way is to use the factors to compensate the difference caused by the PWAS locations by defining

$$
w_{m}=\sqrt{\tilde{r}_{m}}
$$

This yields a beamforming factor of

$$
B F\left(w_{m}, \vec{r}, \vec{s}_{m}, \phi_{0}\right)=\sum_{m=0}^{M-1} 1=M
$$

Recalling Equation (8), we found that the synthetic wave $z(\vec{r}, t)$ is $M$ times reinforced of the wave coming from a single PWAS located at the origin. This manifests that with proper delays and weights, phased arrays are able to direct at certain direction with enhanced magnitude. When the direction $\phi_{0}$ changes, beam steering of the PWAS phased array is accomplished thereby.

\section{2-D PWAS PHASED ARRAY DEVELOPMENT}

The delay formulas developed in previous section is for generic situation, effective for any array configuration. The simplest array that can be implemented is the linear array by arranging all elements along a straight line [5][7][8]. However, 1-D linear array has the intrinsic defect that it cannot distinguish the reflections from two sides (in front or behind the array) due to its geometric symmetry and their view area is limited to $180^{\circ}$ only.

Array elements can be arranged in 2-D planar grid with rectangular or circular shapes [9] [12]. The planar arrays should be more versatile, able to provide beamforming with lower sidelobes, and most importantly, able to scan the main beam of the array toward any point within the $0^{\circ} \sim 360^{\circ}$ range. We will explore and compare the beamforming of different planar arrays. The design and MathCAD beamforming simulation are provided in this section.

\subsection{2-D array designs and simulation}

In this section, the simulation assumed using 1-mm thick aluminum plate and 7-mm round PWAS tuned to 300 
$\mathrm{kHz}$ to obtain an ideal non-dispersive S0 Lamb wave mode with a propagating speed $c=5440 \mathrm{~m} / \mathrm{s}$.

\subsubsection{Cross-shaped array}

The cross-shaped array layout is illustrated in Figure 3a. Cartesian coordinates are used. Array elements are distributed along $x$ - and $y$ - directions, spaced at $d_{x}$ and $d_{y}$, respectively. Beamforming results at various directions using an $M=N=9$ cross-shaped array are shown in Figure $3 \mathrm{~b}$. For $20^{\circ}$ and $60^{\circ}$, unique directional beams are obtained at desired directions, though with significant sidelobes. But for $0^{\circ}$ and $90^{\circ}$, duplicated beams in the opposite direction are also present $\left(180^{\circ}\right.$ for beamforming at $0^{\circ}$, and $270^{\circ}$ for beamforming at $90^{\circ}$ ). Therefore, if using cross-shaped arrays, $360^{\circ}$ full range beamforming is only conditionally attained. For the directions along the array alignments, such as $0^{\circ}, 90^{\circ}, 180^{\circ}$, and $270^{\circ}$, duplicated beams appear.
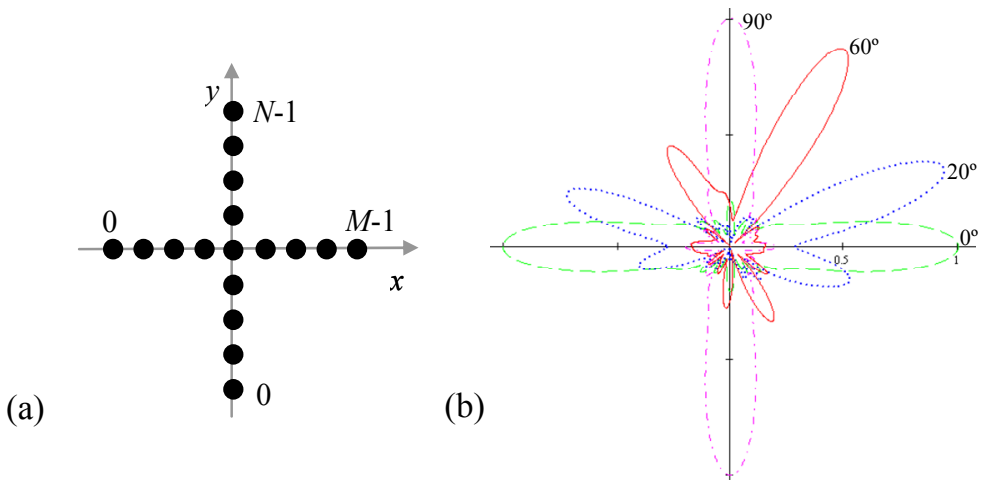

Figure 3 2-D cross-shaped array design. (a) schematic of a cross-shaped array with $M$-element in $x$ direction and $N$-element $y$ direction; (b) beamforming at various angles with $d_{x}=d_{y}=0.5 \lambda, r / d=10, M=N=9$

\subsubsection{Rectangular array}

Rectangular arrays are simply constructed by aligning linear arrays, as illustrated in Figure 4a. Array elements are distributed in the $x$ - and $y$ - directions, spaced at $d_{x}$ and $d_{y}$, respectively. Beamforming at various directions using an $8 \times 8$ PWAS array is shown in Figure 4b. For this array, unique directional beams at all desired directions, $0^{\circ}, 20^{\circ}, 80^{\circ}, 150^{\circ}, 260^{\circ}$, and $330^{\circ}$, have been successfully obtained, with low sidelobes. The simulation results show that a rectangular array is a good candidate for $360^{\circ}$ full-range damage detection. A rectangular array will reduce to a 1-D linear array when either $M$ or $N$ decreases to one. Beamforming at $20^{\circ}$ and $80^{\circ}$ with $N=1$ is given in Figure 4c. The resulting lobes are symmetric about $0^{\circ} / 180^{\circ}$ ( $x$-direction), as expected.

(a)

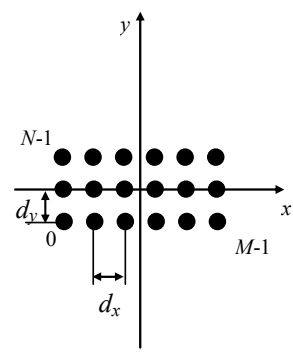

(b)

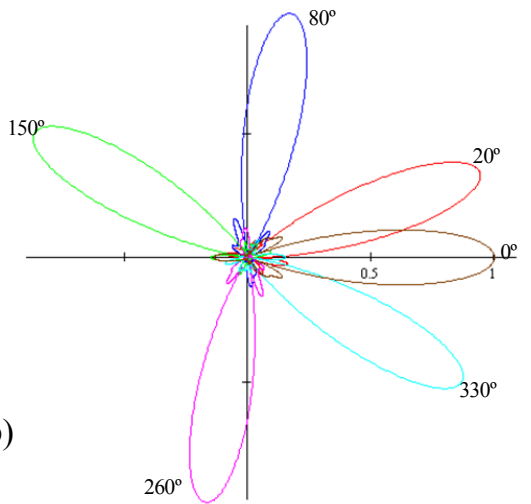

(c)

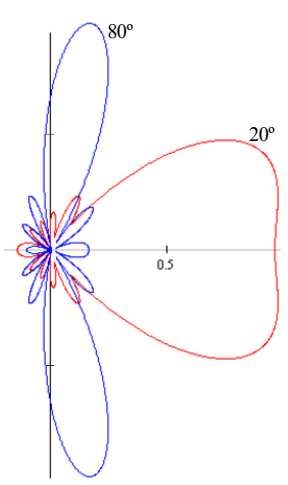

Figure 4 2-D rectangular array design. (a) schematic of an $M \mathrm{x} N$ rectangular array spacing at $d_{x}$ and $d_{y}$; (b) beamforming at various angles with $d_{x}=d_{y}=0.5 \lambda, r / d=10, M=N=8$; (c) beamforming at $20^{\circ}$ and $80^{\circ}$ when $N=1$, with scanning range reduced to $180^{\circ}$ range (two main lobes symmetrical about the horizontal axis)

\subsubsection{Rectangular ring array}

Rectangular arrays have good directionality in the $360^{\circ}$ full range. However, to construct a rectangular array, a large number of PWAS have to be used to cover all the grid nodes, which will results in the complexity of 
wiring and data collection. A substitute for the rectangular array is the rectangular ring array, where only the elements at the circumference are used (Figure 5a). Beamforming at various directions using a rectangular ring array with eight PWAS each side is given in Figure 5b. It can be seen that directional beamforming within the $360^{\circ}$ full range has been obtained. Compared with the equivalent $8 \times 8$ rectangular array, the rectangular ring array obviously has much severe sidelobes while yielding a slightly finer mainlobe.

\subsubsection{Circular ring array}

The circular ring array is shown in Figure 6a, uniformly spaced at $d$. Beamforming at various direction using a 64-PWAS circular ring array spaced at $0.5 \lambda$ is shown in Figure $6 \mathrm{~b}$. Compared with the equivalent 64-PWAS rectangular array (Figure 4b), beamforming of the circular ring array has finer mainlobe (better directivity) but much higher sidelobe levels. Note that the ring array has a diameter of about $163 \mathrm{~mm}$ while the rectangular array has a diagonal of $80 \mathrm{~mm}$, i.e., the equivalent rectangular array has a much compact size. When $M=2$, the circular ring array is reduced to a classic dipole (Figure 6c).

\subsubsection{Concentric circular array}

Concentric circular array is composed of a group of ring arrays with a common center, as shown in Figure 7a. The spacing between neighboring rings is defined as $d_{\mathrm{r}}$ (radial spacing) while the spacing between neighboring elements in the same ring is defined as $d_{\mathrm{c}}$. The radius $R_{n}$ of $n^{\text {th }}$ ring is simply $n^{*} d_{\mathrm{r}}, n=0,1,2 \ldots(N-1)$. The number of elements $M$ in the $n^{\text {th }}$ ring can be determined by rounding up $2 \pi n d_{r} / d_{c}$ to the closest integer. Beamforming result at various directions of a 5-ring concentric circular array is shown in Figure $7 \mathrm{~b}$. The number of elements in each ring is $\{1,7,13,19,26\}$, requiring a total of 66 PWAS. Compared with the 8x8 rectangular array, we see that both arrays have good directionality within the $360^{\circ}$ full range and have similar beamforming performance while the circular array yields a little higher sidelobe levels.

(a)

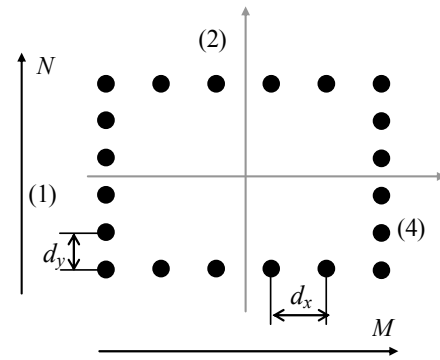

(3)

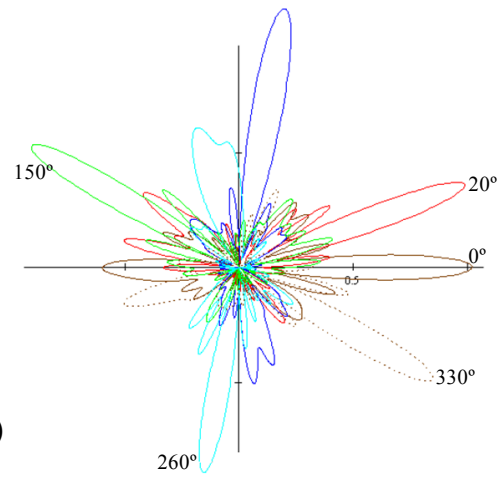

Figure 5 2-D rectangular ring array design. (a) schematic of a rectangular ring array spacing at $d_{x}$ and $d_{y}$; (b) beamforming at various directions for such an array with 8 PWAS on each side, $d_{x}=d_{y}=0.5 \lambda, r / d=10$

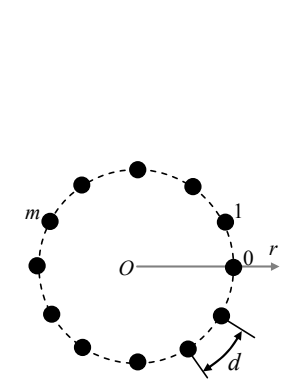

(a)

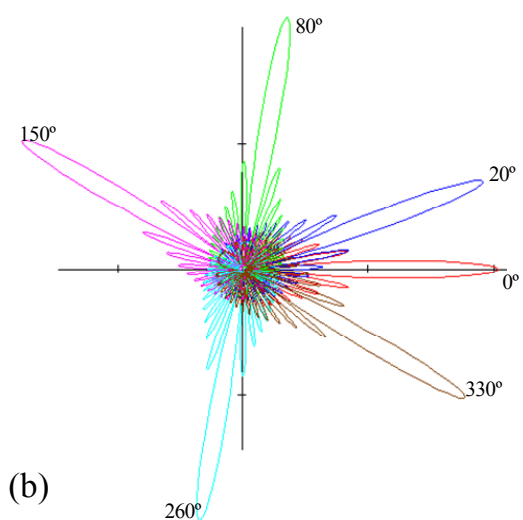

(c)

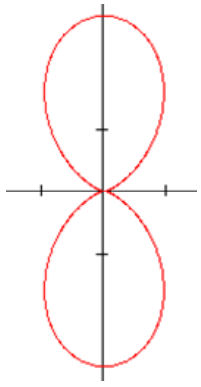

c)

Figure 6 2-D circular ring array design. (a) schematic of the circular ring array; (b) beamforming at various angles with $d=0.5 \lambda$, $r / d=10, M=64$; (c) beamforming when $M=2$ when the circular ring array is reduced to a classic dipole 


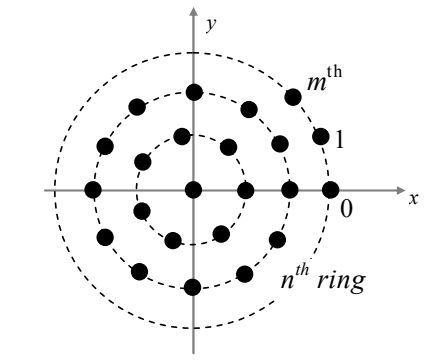

(a) (b)

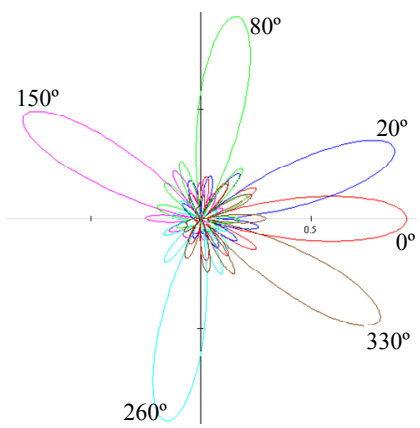

Figure 766 element circular disk array: (a) layout of the circular disk array; (b) beamforming within $0^{\circ} \sim 360^{\circ}$ range at different angles with $d_{\mathrm{c}} / \lambda=d_{\mathrm{r}} / \lambda=0.5 ;$ (c) $M=2$, the circular ring array is reduced to a horizontally arranged dipole

\subsection{2-D PWAS phased array implementation: the embedded ultrasonic structural radar}

For PWAS arrays, the beamforming for sending and receiving signals is implemented through a signal post processing procedure, known as the embedded ultrasonic structural radar (EUSR) algorithm, for in-situ damage detection and structural health monitoring [13]. Preliminary results of using 1-D EUSR can be found at [14][15][16]. For 2-D EUSR, we appled it to an $M \mathrm{x} N$ rectangular array for proof of concept. Different from the 1-D implementation, we need to define the front side of the 2-D arrays. That is to say, we need to establish numbering rule for setting up the order of firing in the arrays. The numbering used in 1-D situation is to start from left to right (such that the front side is determined). Extending to the 2-D situation, the rule of numbering still starts from left to right and then moves from the back to front. By this means, the front side of the array can be determined. If the $0^{\circ}$ direction (or front side of the array) is determined, the numbering of an $8 \times 8$ array will start from the left-bottom corner and increase from left to right (as the arrow indicates). By this means, the crack is positioned at $90^{\circ}$ (Figure 8a).

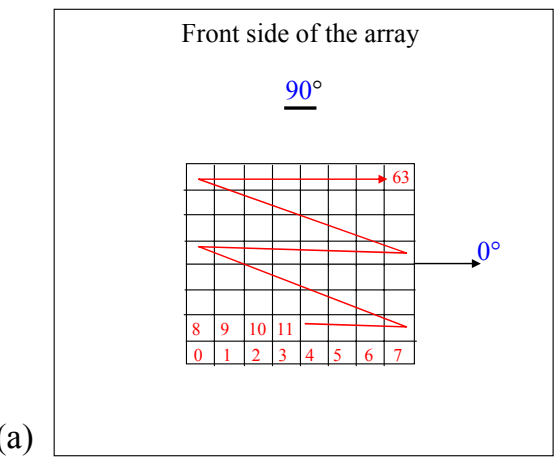

(b)

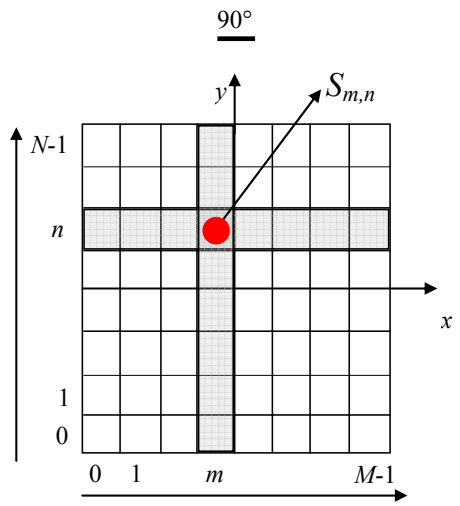

Figure 8 2-D $M \mathrm{x} N$ rectangular PWAS array indexing. (a) array numbering starting from the left bottom, determining the front side of the array; (b) details of the numbering and resulting element coordinates

Data collection in EUSR system is conducted in a round-robin pattern. At each time, one transmitter sends out excitation and the rest serve as receivers to pick up the reflections. Then all the elements in the array serve as transmitters in turn. When the round-robin data collection is done, there will be altogether $M_{\text {total }}^{2}$ signals recorded. After this, the 2-D EUSR algorithm post-processes the data set, implementing the simultaneous firing by applying delays to the sending out signals in virtual time. The same procedure is repeated to obtain reflection beamforming. By changing the target direction $\phi_{0}$, the 2-D EUSR steers the directional beams, both transmission and reflection, sweeping through the specimen and present the scanning results as a 3-D color image. The A-scan signal at a direction dialed-in by the user is also available in the user interface. The transmission or reception beamforming at a direction $\phi_{0}$ after appropriate delays can be expressed as 


$$
z\left(\phi_{0}\right)=\sum_{T}\left\{\sum_{R} f\left(t-\Delta_{T, \phi_{0}}-\Delta_{R, \phi_{0}}\right)\right\}
$$

Or,

$$
z\left(\phi_{0}\right)=\sum_{n_{1}=0}^{N-1} \sum_{m_{1}=0}^{M-1}\left\{\sum_{n_{2}=0}^{N-1} \sum_{m_{2}=0}^{M-1} f\left(t-\Delta_{\left(n_{1}, m_{1}\right), \phi_{0}}-\Delta_{\left(n_{2}, m_{2}\right), \phi_{0}}\right)\right\}
$$

\section{EXPERIMENTS WITH RECTANGULAR ARRAYS}

A proof-of-concept system was built to verify the damage detection ability using various Lamb wave PWAS phased arrays (Figure 9a). DAQ module uses a computer (laptop) to control and collect data from multi-channel data collection equipment, which consists of: (i) a HP33120A arbitrary signal generator; (ii) a Tektronix TDS210 digital oscilloscope; and (iii) a computer controlled auto-switch unit, ASCU unit [17]. The HP33120A was used to generate the excitation with a $10 \mathrm{~Hz}$ repetition rate. The Tektronix TDS210 digital oscilloscope, synchronized with the digital generator, was used to collect the response signals from the PWAS array. The actuation and sensing function of the PWAS elements are automatically controlled by the ASCU unit. 1220-mm (4-ft) square panel of 1-mm (0.040-in) thick 2024-T3 Al-clad aircraft grade sheet metal specimens with different crack layouts were used. The size of the simulated cracks is 19 -mm $(0.750$-in) long, $0.127-\mathrm{mm}(0.005$-in) wide. The PWAS used is APC- 850 small PZT wafer $7 \mathrm{~mm}$ round or square and $0.2 \mathrm{~mm}$ thick. Figure $9 \mathrm{~b}$ shows the layout of the specimens.

In order to minimize the Lamb modes appearing in the wave and strengthen the available mode, a frequency tuning process is established. The excitation frequency has been experimentally selected as $282 \mathrm{kHz}$ at which only S0 mode is maximally excited, avoiding disturbance from dispersion. The data is collected in a round robin pattern resulting in a total of $M^{2}$ data set where $M$ is the number of elements in the array. EUSR algorithm will process the raw data and map the scanning beams in a 2D/3D image for further structural diagnosis.

(a)

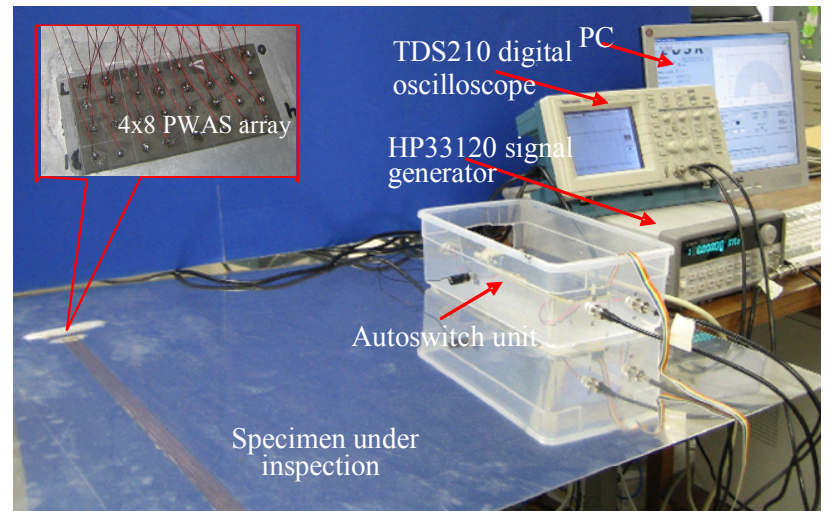

(b)

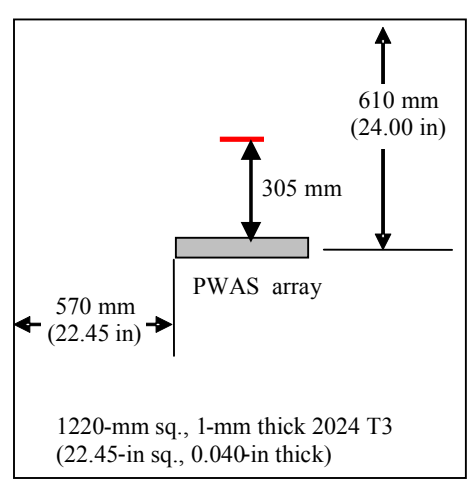

Figure 9 Proof-of-concept EUSR experiment. (a) laboratory experiment setup; (b) specimen layout

\subsection{Preliminary $4 x 8$ rectangular PWAS array EUSR inspection}

For the proof-of-concept, a $4 \times 8$ PWAS rectangular array is first tried. Theoretical beamforming simulation operating at $282 \mathrm{kHz}$ is predicted and shown in Figure 10a. Notice, with this configuration, the theoretical beamforming for $90^{\circ}$ has significant backlobe $\left(270^{\circ}\right)$ though the strongest reflection still comes from $90^{\circ}$. Since the main lobe is much larger than the back lobe, the disturbance due to the back lobe can be removed by setting a threshold value at the backlobe level, under which the values will be forced to zeroes. Original 2-D EUSR scanning result is shown in Figure 10b. An obvious phantom image at $270^{\circ}$ can be seen. Figure $10 \mathrm{c}$ shows the image after the thresholding process to remove the back lobe influence. 


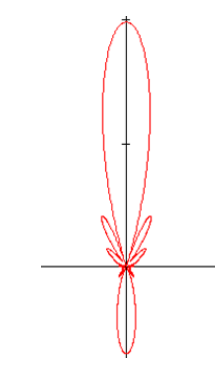

(a)

(b)

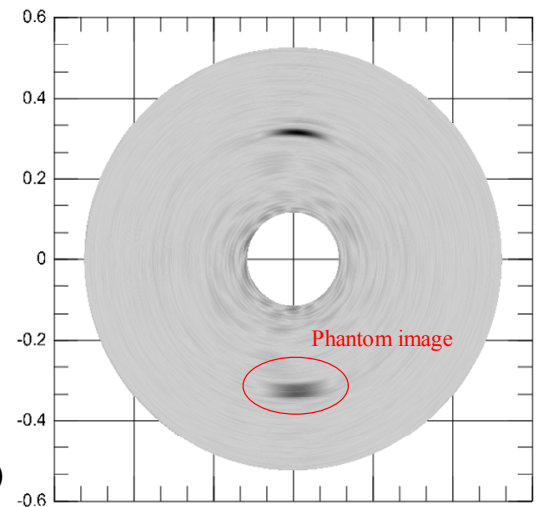

(c)

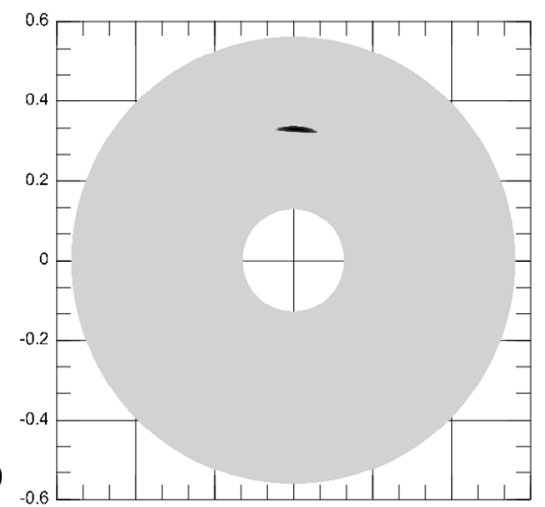

Figure $102 \mathrm{D} 4 \times 8$ rectangular array EUSR inspection. (a) theoretical beamforming at $90^{\circ}$; (b) array numbering method, making the crack appear in front of the array $\left(90^{\circ}\right)$; (c) EUSR inspection image

\subsection{Multiple damage detection using an $8 x 8$ rectangular PWAS array}

One way to improve the performance of the rectangular array is to employ more elements in the array [9]. An $8 \times 8$ array (a total of 64 PWAS) constructed by 7-mm round PWAS is to be used in this section to detect multiple damage situations in aluminum plates (Figure 11a). Specimen layout is illustrated in Figure 11b. Four damage situations will be considered. They are: (1) a single inclined crack at $90^{\circ}$; (2) a single inclined crack at $270^{\circ}$ (by reversing the excitation order); (3) a single pin-hole of 4-mm diameter at $180^{\circ}$; (4) both incline crack at $90^{\circ}$ and 4-mm pin-hole at $270^{\circ}$. Both crack and hole are $305 \mathrm{~mm}$ away from the array center. Theoretical beamforming of the $8 \times 8$ PWAS array operating at $270 \mathrm{kHz}$ tuning frequency is shown in Figure 11c. With the $8 \times 8$ configuration, we see the beamforming is greatly enhanced with much suppressed sidelobe level compared with the $4 \times 8$ configuration. Particularly for beamforming at $90^{\circ}$, no backlobe is observed in the simulation. Laboratory experiments have been performed and the results are given below.

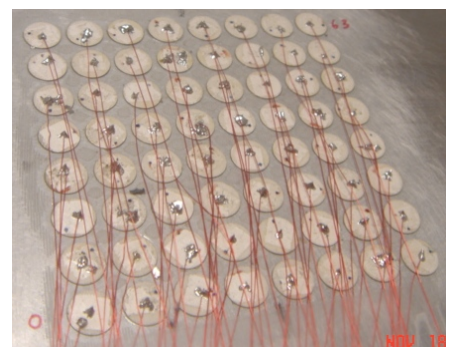

(a)

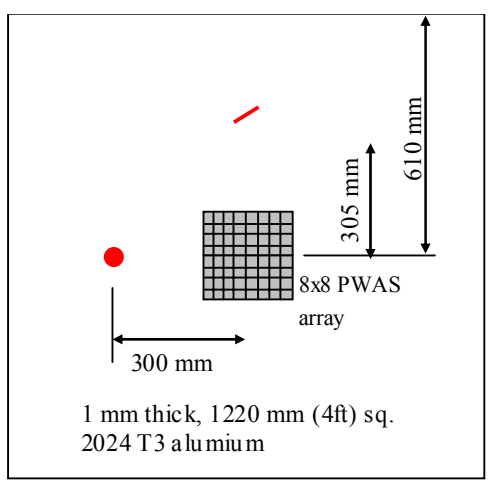

(c)

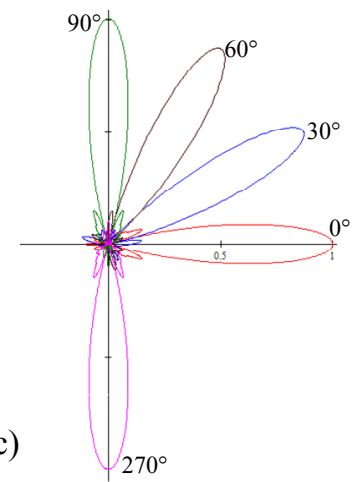

Figure 11 2D 8x8 array damage detection. (a) 8x8 PWAS phased array constructed by 64 7-mm round PWAS; (b) specimen schematic; (c) theoretical beamforming at various directions

\subsection{1. $\underline{8 \times 8 \text { rectangular array: single } 90^{\circ} \text { inclined crack }}$}

The $8 \times 8$ array is first to use to detect a single $90^{\circ}$ inclined crack demonstrated in Figure $11 \mathrm{~b}$. The crack is $45^{\circ}$ inclined to the horizon, otherwise the same as the previous crack. Original scanning result is given in Figure 12a and Figure $12 \mathrm{~b}$ is the image after thresholding. The EUSR image indicates a defect at $90^{\circ}$. Note that the crack image is weaker compared with that obtained by the $4 \times 8$ array. This is due to the reason that we can not get specular reflection since the crack in this specimen is inclined (Figure 11a). For the 8x8 array, the strongest reflection was found to be in $95^{\circ}$, slightly away from right front side. Figure $12 \mathrm{c}$ shows the A-scan segment at $95^{\circ}$ with the thresholding level being used for the improved image. 


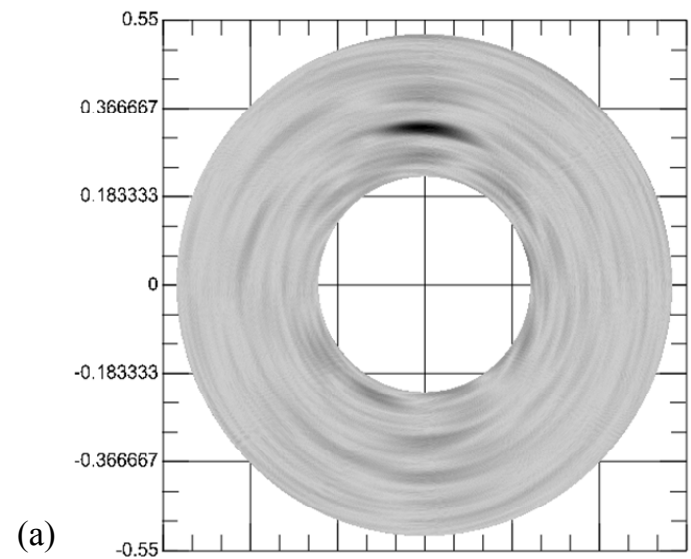

(b)

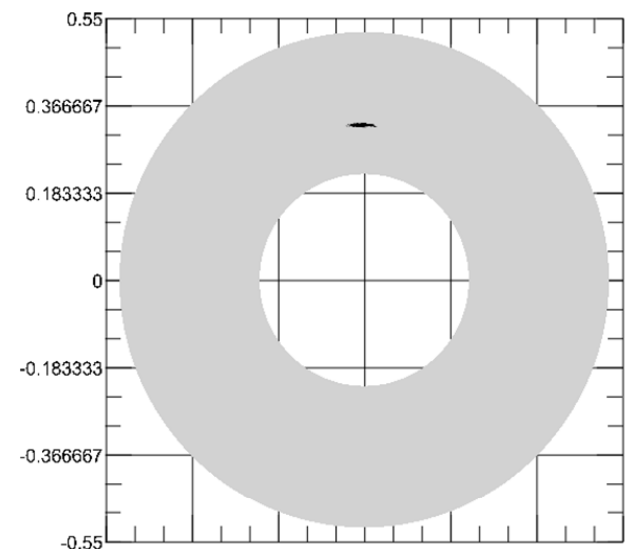

(c)

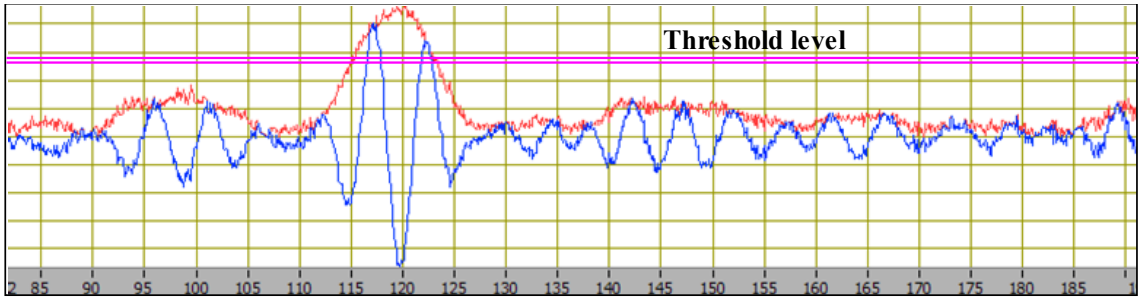

Figure 12 2-D 8x8 PWAS array for single inclined crack at 90 . (a) original EUSR image; (b) threshold EUSR image; (c) A-scan at $95^{\circ}$ and image thresholding level

\subsection{2. $\quad 8 \times 8$ rectangular array: single $270^{\circ}$ inclined crack}

The crack will be located at the back of the array $\left(270^{\circ}\right)$ if the excitation order is reversed. Original scanning image is given in Figure 13a, indicating a strong reflection at $270^{\circ}$. For this situation, the strongest reflection was found to be in $-85^{\circ}$, slightly away from $270^{\circ}$. Figure $13 \mathrm{~b}$ shows the improved image after the thresholding process. With the first two experiments, we successfully proved that 2-D EUSR PWAS array has $360^{\circ}$ full range damage detection ability and can correctly located the defect.

(a)

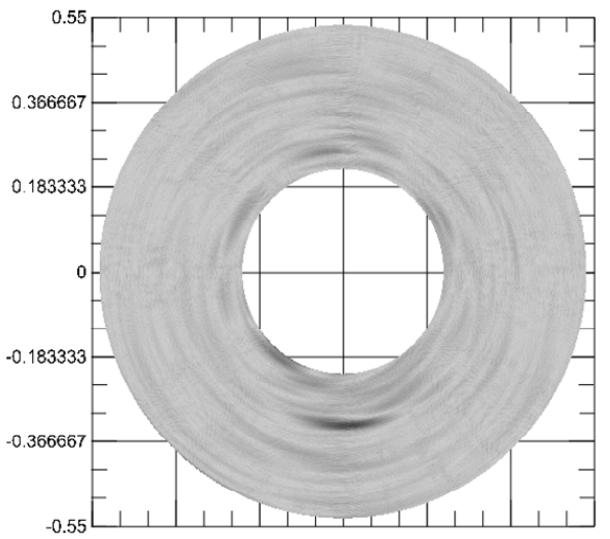

(b)

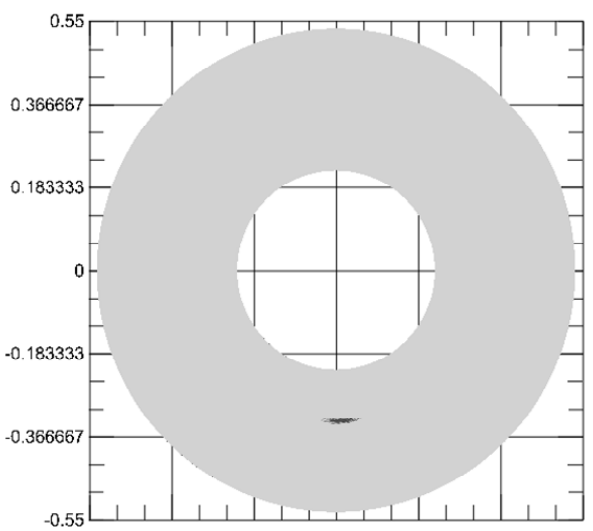

Figure 13 2-D 8x8 PWAS array for single inclined crack at $90^{\circ}$. (a) original EUSR image; (b) threshold EUSR image

\subsection{3. $8 \times 8$ rectangular array: single $180^{\circ}$ hole}

A plate with a single pin hole with a diameter of $4 \mathrm{~mm}$ at $180^{\circ}$ is being used. After thresholding process, a small reflection caused by the pin-hole is observed in Figure 14a at target direction $180^{\circ}$. This experiment verifies that EUSR is able to pick small defect such as a pin-hole.

\subsection{4. $8 \times 8$ rectangular array: inclined crack at $90^{\circ}$ and pin-hole at $180^{\circ}$}

A more complicated specimen is designed to have two defects in the plate, the inclined crack at $90^{\circ}$ and the 4- 
$\mathrm{mm}$ pin hole at $180^{\circ}$, both being $305 \mathrm{~mm}$ away from the PWAS center. EUSR scanning images are presented in Figure 14b. By comparison, the reflection caused by the crack is much stronger than that from the pin-hole. However, EUSR not only detected both but also demonstrated the difference between different defect types clearly.

(a)

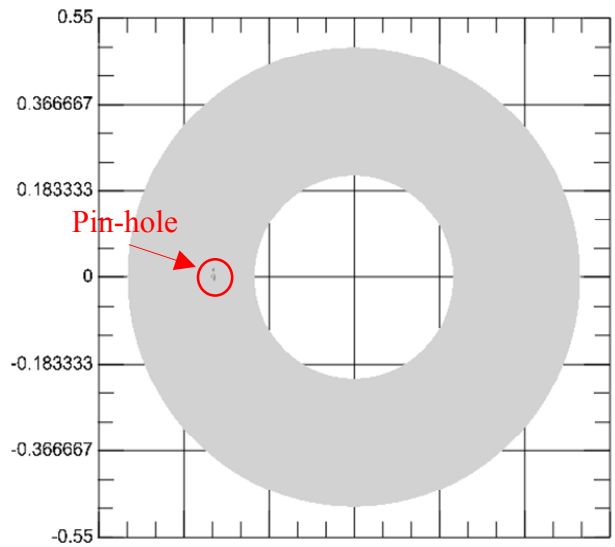

(b)

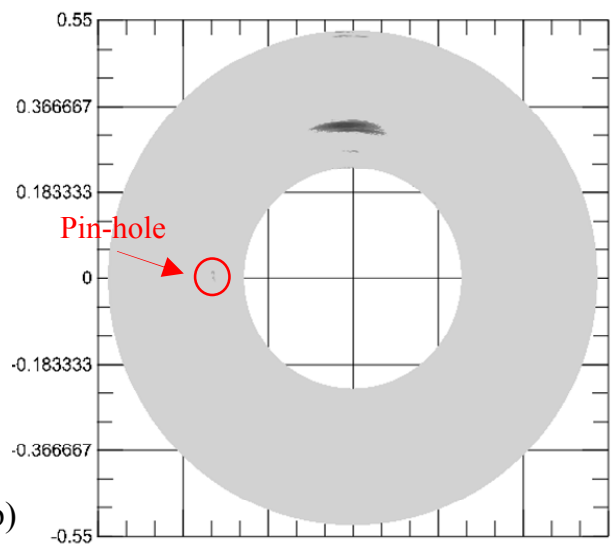

Figure 14 2-D EUSR images of using an 8x8 PWAS array for single pin hole and hole-and-crack situations. (a) a single $180^{\circ} 2$ $\mathrm{mm}$ pin hole (b) $90^{\circ}$ inclined crack and $180^{\circ} 2-\mathrm{mm}$ pin hole

\section{CONCLUSIONS}

In this paper, an in-situ method for detecting damage in thin-wall structures using two-dimensional (2-D) embedded phased-arrays of piezoelectric wafers active sensors (PWAS) has been described. This method uses scanning beams of guided waves (e.g., Lamb wave and Rayleigh wave in plates) that can travel at large distances with little energy loss and hence permit the interrogation of large structural areas from a single location. The present concept is an emerging in-situ Lamb wave inspection technology that is different from conventional ultrasonic phased-arrays because it uses unobtrusive and inexpensive permanently-attached PWAS to perform on-demand structural interrogation. The PWAS phased arrays rely on the selective excitation of single-mode guided-waves that are best adapted for phased-array damage detection for a given plate thickness and material characteristics. Most importantly, different from the conventional ultrasonic phased array, PWAS array scanning is implemented in virtual time through signal post-processing methods, known as the embedded ultrasonic structural radar (EUSR) algorithm. The unique virtual beamforming not only avoids the necessity of using complex electronics but also make the algorithm easy to integrate with more advanced signal processing methods for better scanning performance.

To verify the analysis and evaluate the practical applicability of the 2-D PWAS phased array concept, rectangular $4 \times 8$ and $8 \times 8$ planar PWAS arrays have been bonded on large aluminum plates and used to detect small cracks seeded into the plate. The $4 \times 8$ array proved its full-field scanning capabilities by correctly imaging the small crack in the 3600 domain. However, an issue that we had to confront during the $4 \times 8$ PWAS phased array experiments was the phantom image caused by the backlobe. To overcome the limitation caused by the inequality of the number of elements in different direction, an 8x8 PWAS array was also investigated. Compared with the EUSR image obtained from the $4 \times 8$ array, the $8 \times 8$ array demonstrates improved full range damage detection ability, with no phantom image in other directions. However, since we can not get spatula reflection from inclined crack, the crack image was weaker than that obtained by the $4 \times 8$ array and the strongest A-scan signal was found to be in $95^{\circ}$ direction. Additionally, to further prove EUSR sensitivity to different defect types, experiments involving pin-holes were conducted. The results verified that EUSR is able to detect the crack, the pin-hole, and both.

In summary, the potential of 2-D PWAS phased array for full range damage detection in NDE/SHM applications was demonstrated. We will further research the application of 2-D PWAS arrays in other configurations and make the object of future publications on this subject.

\section{ACKNOWLEDGMENTS}

This material is based upon work supported by the National Science Foundation under Grant \#CMS-0408578 
and \#CMS-0528873 and by the Air Force Office of Scientific Research under Grant \# FA9550-04-0085.

\section{REFERENCES}

[1] Giurgiutiu, V. (2006) "In-situ Structural Health Monitoring, Diagnostics and Prognostics System Utilizing Thin Piezoelectric Sensors", US Patent, Patent No. US7024

[2] Giurgiutiu, V. (2005), "Tuned Lamb Wave Excitation and Detection with Piezoelectric Wafer Active Sensors for Structural Health Monitoring”, Journal of Intelligent Material Systems and Structures, Vo. 16, April 2005, pp. $291 \sim 305$

[3] Bottai, G.; Giurgiutiu, V. (2005) "Simulation of the Lamb Wave Interaction between Piezoelectric Wafer Active Sensors and Host Structure”, SPIE volume 5765 (2005), paper \# 5765-29

[4] Johnson, D. H. and Dudgeon, D. E. (1993), Array Signal Processing: Concepts and Techniques, PTR Prentice Hall, Upper Saddle River, NJ 07458

[5] Clay, A. C.; Wooh, S. C.; Azar, L.; and Wang, J. Y. (1999) "Experimental Study of Phased Array Beam Steering Characteristics", Journal of Nondestructive Evaluation, Vol. 18, No. 2, 1999, pp. 59 71

[6] Deutsch, W.; A.K, Cheng, A.; Achenbach, J. D., "Defect Detection with Rayleigh and Lamb Waves Generated by a Self-Focusing Phased Array", NDT.net, December, Vol. 3 No. 12, March 1998

[7] Purekar, A. S.; Pines, D. J.; Sundararaman, S.; and Adams, D. E. (2004) "Directional Piezoelectric Phased Array Filters for Detecting Damage in Isotropic Plates", Journal of Smart Materials and Structures, Vol. 13, 2005, pp. 838 850

[8] Sundararaman, S. and Adams, D. E. (2002) "Phased Transducer Arrays for Structural Diagnostics Through Beamforming", Proceedings of the 17th Technical Conference of the American for Composites, West Lafayette, IN, Oct. 21 23, 2002, Paper \# 177

[9] Balanis, C.A. (2005), Antenna Theory, John Wiley \& Sons, Inc., Hoboken, NJ 07030

[10] Wilcox, P. (2003) “Omni-directional Guided Wave Transducer Arrays for the Rapid Inspection of Large Areas of Plate Structures”, IEEE Transaction on Ultrasonics, Ferroelectrics, and Frequency Control, Vol. 50, No. 6, June 2003, pp. 699

[11] Wilcox, P.; Lowe, M.; Cawley, P. (2005) “Omnidirectional Guided Wave Inspection of Large Metallic Plate Structures Using an EMAT Array", IEEE Transactions on Ultrasonics, Ferroelectrics, and Frequency Control, Vol. 52, No. 4, April 2005, pp. 653 665

[12] Fromme, P.; Wilcox, P.; Lowe, M.; and Cawley, P. (2005) “A Guided Ultrasonic Waves Array For Structural Integrity Monitoring”, Quantitative Nondestructive Evaluation, Vol. 24, pp. 1780 1787

[13] Giurgiutiu, V.; Bao, J.; Zagrai, A.N. (2006) "Structural Health Monitoring System Utilizing Guided Lamb Waves Embedded Ultrasonic Structural Radar”, U.S. Patent, Patent No. US6996480B2, Feb, 2006

[14] Yu, L.; Giurgiutiu, V. (2005) "Multi-damage Detection with Embedded Ultrasonic Structural Radar Algorithm using Piezoelectric Wafer Active Sensors through Advanced Signal Processing", SPIE volume 5788 (2005), paper \# 5768-48

[15] Yu, L. and Giurgiutiu, V. (2005), "Using Phased Array Technology And Embedded Ultrasonic Structural Radar For Active Structural Health Monitoring And Nondestructive Evaluation", Proceedings of 2005 ASME International Mechanical Engineering Congress, November 5-13, Orlando, FL, paper \# IMECE2005-80227

[16] Yu, L. and Giurgiutiu, V., "Improvement of Damage Detection with the Embedded Ultrasonics Structural Radar for Structural Health Monitoring", Proceedings of $5^{\text {th }}$ International Workshop on Structural Health Monitoring, Stanford University, Stanford, CA, September 15-17, 2005

[17] Liu, W.; Giurgiutiu, V. (2005) "Signal Acquisition/Conditioning for Automated Data Collection during Structural Health Monitoring with Piezoelectric Wafer Active Sensors", Proceedings of the 5th International Workshop on Structural Health Monitoring, 11-14 September 2005, Stanford University, CA, pp. 606-618 